\title{
APPROXIMATION OF THE ARCH PROBLEM BY RESIDUAL-FREE BUBBLES
}

\author{
A. Agouzal ${ }^{1}$ And M. El Alami El Ferricha ${ }^{2}$
}

\begin{abstract}
We consider a general loaded arch problem with a small thickness. To approximate the solution of this problem, a conforming mixed finite element method which takes into account an approximation of the middle line of the arch is given. But for a very small thickness such a method gives poor error bounds. the conforming Galerkin method is then enriched with residual-free bubble functions.
\end{abstract}

Résumé. On considère un problème de déplacement d'une arche chargée et de faible épaisseur. Pour approcher la solution de ce problème, on donne une méthode d'éléments finis Galerkin mixte conforme qui tient compte d'une approximation de la forme de l'arche. Cependant une application directe d'une telle méthode ne donne pas de résultat de convergence satisfaisant pour une faible épaisseur. On propose d'enrichir cette méthode par des fonctions bulles résiduelles.

Mathematics Subject Classification. 65N12.

Received: March 13, 2000. Revised: November 29, 2000.

\section{INTRODUCTION}

We are interested with a numerical analysis of a general arch problem with a small thickness. The elastic, clamped and loaded arch is studied by following the Budiansky-Sander's model in linearized elasticity. The variable considered herein is the displacement of the arch under the potential energy of exterior forces. The shape of (the middle line) of the arch is assumed to be represented by a smooth function $\Phi$. The coefficients, in the state equation governing this problem, depend on $\Phi$ and on its third derivative. Following Lods [18] we give a mixed formulation that is equivalent to the state equation in which the primitive variables are the components of the displacement vector in a fixed basis, the rotation of the normal vector and the membrane energy. The coefficients of this reformulated problem only depend on $\Phi$ and on its first derivative. We explicitate the dependence of these coefficients on a critical parameter that is the thickness of the arch. The resulting problem fit into a singularly perturbed one. For the discrete problem, following Bernadou-Ducatel [5], we approach the arch by straight beam elements of small length. But, when the thickness is very small, numerical results obtained from commonly used standard finite element method, deteriorate. Interesting study of this phenomenon has been performed by Habbal and Chenais in [17] where it is proved by a non-conforming finite elements that if the mesh size is taking in the order of the square of the thickness, the convergence is insured. We may also mention that, a precise mathematical meanings for locking and robustness phenomenon are giving by Babuska

\footnotetext{
Keywords and phrases. Mixed method, Lagrange multipliers, conforming approximations, residual-free-bubble.

1 Université Lyon 1, L.A.N., bâtiment 101, boulevard du 11 novembre 1918, 69622 Villeurbanne Cedex, France.

2 École Normale Supérieure, Fès, Département de Mathématiques et Informatique, BP 5206, Bensouda, Fès, Morocco.

e-mail: elferrida@caramail.com
} 
and Suri in [3]. To complete we notice that a study of the numerical behaviour is performed in [12] by Chenais and Paumier for a class of elliptic problem in which a small parameter is involved, the computation of elastic arches is analyzed using this mathematical framework and general conditions ensuring uniform convergence of a numerical scheme, with respect to the thickness, are given.

In this paper, we consider the finite element method where the finite-dimensional space used consists of piecewise polynomials enriched with bubble functions (see [4,16,21,22]). More precisely our computed solution is the sum of a piecewise polynomial and a bubble function that vanishes on the boundary of each element. We shall concentrate on the variant of this method where the bubbles are residual-free (see [21,22]). For piecewise linears considered the bubbles are exactly computed. Since these functions vanish on the boundary of each element, they can be eliminated or statically condensed. The discrete problem is finally written in terms of the classical polynomial-based test space.

The structure of the paper is as follows: in the next section we consider the continuous arch problem. We introduce a mixed formulation with Lagrange multipliers where the new unknowns are considered. Following Lods [18] we establish that this mixed formulation is equivalent to the usual state equation governing the arch problem. In the remainder of this section, we consider $\varepsilon$, the thickness of the arch, as a small parameter of perturbation. We explicitate the dependence of the coefficients of the variational formulation on $\varepsilon$, we then concentrate on the resulting perturbed problem for which the key conditions of Brezzi's theorem are given. In Section 3 we first consider the discrete problem obtained from a direct application of the Galerkin method and from an approximation by interpolation of the arch. Consistency error estimates are proved. The stability conditions, with associated constants depending on $\varepsilon$, are then established. Some error bounds are given. Thereafter we introduce the residual-free bubbles technique, we calculate the bubble functions associated to piecewise linears, then we use the static condensation procedure to obtain the new discrete problem. Finally, we report on some numerical simulations that illustrate the suitability of the method with bubbles presented herein.

\section{The CONTINUOUs PROBlem}

\subsection{The state equation}

The shape of the arch is represented by a function $\Phi$ of the space:

$$
\Lambda_{s}=\left\{\Phi \in W^{s+1, \infty}(I) \text { such that } \Phi(0)=\Phi(l)=0\right\}
$$

where $l$ is the depth of the arch, $I=[0, l]$ and $s \geq 0$ an integer. If $L$ is the length of the arch and $e$ its thickness, the mean surface is:

$$
\omega=\left\{(x, y, z) \in \mathbb{R}^{3}, x \in I, y=\Phi(x) \text { and } z \in\right] 0, L[\} .
$$

If $\vec{n}(m)$ denotes the unit normal to the surface $\omega$ at the point $m$, the arch is the domain:

$$
\Omega=\left\{m+x_{3} \vec{n}(m), m \in \omega ;-\frac{e}{2}<x_{3}<\frac{e}{2}\right\} .
$$

In the setting of Budiansky-Sanders model in linear elasticity theory (see [10]), the displacement at a point $M$ of $\Omega$ is entirely determined by the displacement at a point $m$ of the mean surface. Since the loading is invariant on the $o z$-axis, the displacement vector belongs to the $(x, y)$-plane. The problem is then reduced to a one dimensional problem. The displacement of a point $(x, \Phi(x))$ is marked by its pair of tangential and normal components in the local basis $(\vec{t}(\Phi), \vec{n}(\Phi))$ :

$$
u(x)=u_{1}(x) \vec{t}(\Phi)(x)+u_{2}(x) \vec{n}(\Phi)(x) .
$$


Where, with $\left(\vec{e}_{1}, \vec{e}_{2}\right)$ the canonical basis of $\mathbb{R}^{2}, \Phi^{\prime}=\frac{\mathrm{d} \Phi}{\mathrm{d} x}$ and $S(\Phi)=\sqrt{1+\Phi^{\prime 2}}$

$$
\vec{t}(\Phi)=\frac{1}{S(\Phi)}\left(\vec{e}_{1}+\Phi^{\prime} \vec{e}_{2}\right) \quad \vec{n}(\Phi)=\frac{1}{S(\Phi)}\left(-\Phi^{\prime} \vec{e}_{1}+\vec{e}_{2}\right)
$$

The arch being clamped, the pair $u=\left(u_{1}, u_{2}\right)$ of components belongs to the admissible displacement space (see $[18])$

$$
W=H_{0}^{1}(I) \times H_{0}^{2}(I)
$$

where $H_{0}^{1}(I)$ and $H_{0}^{2}(I)$ are usual Sobolev spaces. Naturally the displacement $u$ depends on the function $\Phi$. From the virtual work principle $u$ satisfies the state equation (see [5]):

$$
u \in W \quad c(\Phi ; u, v)=l(\Phi ; v)
$$

where the energy $c$ of the arch is given by:

$$
c(\Phi ; u ; v)=E e l \int_{0}^{l} \eta(\Phi ; u) \eta(\Phi ; v) s(\Phi) \mathrm{d} x+\frac{E e^{3} l}{12} \int_{0}^{l} k(\Phi ; u) k(\Phi ; v) s(\Phi) \mathrm{d} x
$$

with $E$ : the Young modulus, $\eta$ : the membrane energy and $k$ : the bending energy, respectively given by:

$$
\eta(\Phi ; v)=\frac{1}{S(\Phi)} v_{1}^{\prime}+\frac{1}{R(\Phi)} v_{2} ; \quad k(\Phi ; v)=\frac{(\theta(\Phi ; v))^{\prime}}{S(\Phi)}
$$

the curvature $\frac{1}{R(\Phi)}$ of $\omega$ and the rotation of the normal vector $\theta(\Phi ; v)$ are defined by:

$$
\frac{1}{R(\Phi)}=-\frac{\Phi^{\prime \prime}}{S(\Phi)^{3}} \quad \text { and } \quad \theta(\Phi ; v)=\frac{v_{1}}{R(\Phi)}-\frac{v_{2}^{\prime}}{S(\Phi)}
$$

The virtual work of exterior load is(with $\rho$ the density of the material):

$$
l(\Phi, v)=-\int_{0}^{l} \rho e\left(\Phi^{\prime} v_{1}+v_{2}\right) \mathrm{d} x .
$$

\subsection{The mixed continuous formulation}

We fix notation and functional setting that shall be used in the analysis below.

Let $(\beta, \gamma)$ denote the pair of components of the displacement vector in the fixed basis $\left(e_{1}, e_{2}\right)$. Designate by $\theta$ and $\eta$ respectively the rotation of the normal vector and the membrane energy; the new unknown vector is: $(\beta, \gamma, \theta, \eta) \in\left(H_{0}^{1}(I)\right)^{3} \times L^{2}(I)$. Let $u=(\beta, \gamma, \theta, \eta)$ be the primal unknown and $v=(\underline{\beta}, \underline{\gamma}, \underline{\theta}, \underline{\eta})$ a generic variable corresponding to $u$. Denote by $p=\left(p_{1}, p_{2}\right)$ the unknown in the space of Lagrange multipliers and $q=\left(q_{1}, q_{2}\right)$ a generic variable associated. We employ the the conventional spaces $L^{2}(\Omega), H^{2}(\Omega), H_{0}^{2}(\Omega)$ and $W^{s, \infty}(\Omega)$ with their classical norms: $\|\cdot\|_{0, \Omega}=|\cdot|_{0, \Omega},\|\cdot\|_{s, \Omega}=\sum_{k=0}^{s}|\cdot|_{k, \Omega}$ and $\|\cdot\|_{s, \infty, \Omega}$; as usual $(a, b)_{\Omega}=\int_{\Omega} a b \mathrm{~d} x$. For brevity we drop the subscript $\Omega$ when $\Omega=I$. Finally we introduce the spaces $V=\left(H_{0}^{1}(I)\right)^{3} \times L^{2}(I)$ and $\sum=L^{2}(I) \times L^{2}(I)$ equipped respectively of the norms:

$$
\begin{aligned}
\|v\|_{V}^{2} & =\|\underline{\beta}\|_{1}^{2}+\|\underline{\gamma}\|_{1}^{2}+\|\underline{\theta}\|_{1}^{2}+\|\underline{\eta}\|_{0}^{2} \\
\|q\|_{\Sigma}^{2} & =\left\|q_{1}\right\|_{0}^{2}+\left\|q_{2}\right\|_{0}^{2} .
\end{aligned}
$$

Now, let us recall some results given by Lods in [18]. 
Proposition 1. Assume that $\Phi \in \Lambda_{s}$. Let $v=\left(v_{1}, v_{2}\right)$ belong to the space $W$ then the following equalities hold in $L^{2}(I)$ :

$$
\begin{aligned}
& \beta^{\prime}=\theta \Phi^{\prime}+\eta \\
& \gamma^{\prime}=-\theta+\Phi^{\prime} \gamma^{\prime}
\end{aligned}
$$

where $\beta \vec{e}_{1}+\gamma \vec{e}_{2}=v_{1} \vec{t}(\Phi)+v_{2} \vec{n}(\Phi), \theta=\theta(v ; \Phi)$ and $\eta=\eta(v ; \Phi)$

Variational formulation. Define the subspace

$$
K(\Phi)=\{v \in V \text { such that } b(\Phi ; v, \mu)=0 \quad \forall \mu \in \Sigma\}
$$

where the continuous bilinear form $b(\Phi ; .,$.$) is given by:$

$$
b(v, \mu)=\int_{0}^{l} \mu_{1}\left(\underline{\beta}^{\prime}-\underline{\theta} \Phi^{\prime}-\underline{\eta}\right) \mathrm{d} x+\int_{0}^{l} \mu_{2}\left(\underline{\gamma}^{\prime}-\underline{\theta}-\Phi^{\prime} \underline{\eta}\right) \mathrm{d} x \quad \forall \mu=\left(\mu_{1}, \mu_{2}\right) \in \Sigma
$$

then we have:

Proposition 2. Assume that $\Phi \in \Lambda_{s}$, then the mapping:

$$
\begin{aligned}
& F^{\Phi}: W \quad \longrightarrow K(\Phi) \\
& v=\left(v_{1}, v_{2}\right) \longmapsto(\underline{\beta}, \underline{\gamma}, \underline{\theta}, \underline{\eta})
\end{aligned}
$$

where $\underline{\beta}=\underline{\beta}(\Phi ; v)$ and $\underline{\gamma}=\underline{\gamma}(\Phi, v)$ is an isomorphism.

By using the isomorphism $F^{\Phi}$, the state equation (2) is equivalent to the following problem (see [18]): find $u=(\beta, \gamma, \theta, \eta) \in K(\Phi)$ such that:

$$
a(\Phi ; u, v)=l(\Phi ; v), \quad \forall v \in K(\Phi)
$$

where the bilinear form $a(\Phi ; .,$.$) and the linear form l(\Phi ;$.$) are respectively given by$

$$
\begin{aligned}
a(u, v) & =E e l \int_{0}^{l} \underline{\eta} \underline{\underline{\eta}} S(\Phi) \mathrm{d} x+\frac{E e^{3} l}{12} \int_{0}^{l} \theta^{\prime} \underline{\theta^{\prime}} \cdot \frac{1}{S(\Phi)} \mathrm{d} x \\
l(v) & =-\rho e \int_{0}^{l} \underline{\gamma} S(\Phi) \mathrm{d} x .
\end{aligned}
$$

By standard arguments, we can set the variational problem with Lagrange multipliers as: find $u=(\beta, \gamma, \theta, \eta) \in$ $V$ and $p=\left(p_{1}, p_{2}\right) \in \Sigma$ such that:

$$
(P) \begin{cases}a(\Phi ; u, v)+b(\Phi ; v, p)=l(\Phi ; v) & \forall v=(\underline{\beta}, \underline{\gamma}, \underline{\theta}, \underline{\eta}) \in V \\ b(\Phi ; u, q)=0 & \forall q=\left(q_{1}, q_{2}\right) \in \Sigma\end{cases}
$$

where the bilinear form $b(\Phi ; .,$.$) is given by$

$$
b(v, q)=\int_{0}^{l} q_{1}\left(\underline{\beta}^{\prime}-\underline{\theta} \Phi^{\prime}-\underline{\eta}\right) \mathrm{d} x+\int_{0}^{l} q_{2}\left(\underline{\gamma}^{\prime}-\underline{\theta}-\Phi^{\prime} \underline{\eta}\right) \mathrm{d} x .
$$


Now, denoting by $A$ and $B$ the operators associated to the forms $a(.,$.$) and b(.,$.$) , we obtain from problem (P)$ the following Euler Lagrange equations:

$$
\begin{aligned}
A u+B^{\star} p & =f \\
B u & =0
\end{aligned}
$$

with:

$$
A u=\left(\begin{array}{c}
0 \\
0 \\
-\frac{E e^{3} l}{12}\left(\frac{\theta^{\prime}}{s(\Phi)}\right)^{\prime} \\
\operatorname{Eels}(\Phi) \eta
\end{array}\right) ; \quad B^{\star} p=\left(\begin{array}{c}
-p_{1}^{\prime} \\
-p_{2}^{\prime} \\
-p_{1} \Phi^{\prime}+p_{2} \\
-p_{1}-\Phi^{\prime} p_{2}
\end{array}\right) ; \quad f=\left(\begin{array}{c}
0 \\
e \rho s(\Phi) \\
0 \\
0
\end{array}\right)
$$

and

$$
B u=\left(\begin{array}{c}
\beta^{\prime}-\theta \Phi^{\prime}-\eta \\
\gamma^{\prime}+\theta-\Phi^{\prime} \eta
\end{array}\right)
$$

The subsequent theorem establishes the equivalence between the mixed problem and the state equation (2), we have (see [18]).

Theorem 3. Assume that $\Phi \in \Lambda_{s}$, then problem $(P)$ admits a unique solution $(u, p)$ and $\underline{u}=\left(F^{-\Phi}\right) u$ is the solution of the state equation.

\subsection{The perturbed problem}

Explicitating the dependence of the coefficients in equations of problem $(P)$ on a small parameter $\varepsilon=\frac{e}{l}$ (the rigidity), taking, for simplicity, $l=1$ and dividing by $E$ the two members of its first equation, problem $(P)$ becomes:

$$
\left(P_{\varepsilon}\right) \begin{cases}a_{\varepsilon}(w, \tau)+b(\tau, p)=l(\tau) & \forall \tau \in V \\ b(w, q)=0 & \forall q \in \Sigma\end{cases}
$$

where

$$
\begin{gathered}
a_{\varepsilon}(w, \tau)=\varepsilon \int_{0}^{1} \eta \underline{\eta} S(\Phi) \mathrm{d} t+\frac{\varepsilon^{3}}{12} \int_{0}^{1} \theta^{\prime} \underline{\theta^{\prime}} \cdot \frac{1}{S(\Phi)} \mathrm{d} t \\
b(\tau, q)=\frac{1}{E l}\left\{\left(\underline{\beta^{\prime}}-\underline{\theta} \Phi^{\prime}-\underline{\eta}, q_{1}\right)_{0}+\left(\underline{\gamma^{\prime}}+\underline{\theta}-\Phi^{\prime} \underline{\eta}, q_{2}\right)_{0}\right\}
\end{gathered}
$$

and

$$
l(\tau)=-\rho \varepsilon(\underline{\gamma}, S(\Phi))_{0, I}
$$

with $I=(0,1)$. To examine existence and uniqueness of solution for $\left(P_{\varepsilon}\right)$, the strategy is to fulfill the two key stability conditions: $(K-$ ellipticity and LBB) (see [2] and [6]);

$$
\left(S_{2}\right) \quad \sup _{\tau \in V} \frac{b(\tau, q)}{\|\tau\|_{V}} \geq C_{2}\|q\|_{\Sigma}^{2} \quad \forall q \in \Sigma
$$


this has been done in the proof of the previous theorem. We observe that for $\varepsilon>0$ the form $a(.,$.$) is K$-elliptic and the problem is well-posed. However for $\varepsilon=0$ problem $\left(P_{0}\right)$ is not solvable. From now on, we shall deal with problem $\left(P_{\varepsilon}\right)$ for $0 \prec \varepsilon \ll 1$ (the numerically more delicate case) which can be written as:

$$
\begin{cases}a_{\varepsilon}(w, \tau)+b_{\varepsilon}(\tau, p)=l(\tau) & \forall \tau \in V \\ b_{\varepsilon}(w, q)=0 & \forall q \in \Sigma\end{cases}
$$

where:

$$
\begin{aligned}
& a_{\varepsilon}(w, \tau)=\int_{0}^{1} \eta \underline{\eta} s(\Phi) \mathrm{d} t+\frac{1}{12} \varepsilon^{2} \int_{0}^{1} \theta^{\prime} \underline{\theta}^{\prime} \cdot \frac{1}{s(\theta)} \mathrm{d} t \\
& b_{\varepsilon}(\tau, q)=\frac{1}{\varepsilon E}\left\{\left(\underline{\beta}^{\prime}-\underline{\theta} \Phi^{\prime}-\underline{\eta}, q_{1}\right)+\left(\underline{\gamma}^{\prime}+\underline{\theta}-\Phi^{\prime} \underline{\eta}, q_{2}\right)\right\}
\end{aligned}
$$

and

$$
l(\tau)=-\rho \int_{0}^{1} \underline{\gamma} S(\Phi) \mathrm{d} t .
$$

We now only explicitate the dependence on $\varepsilon$ of the constants $C_{1}$ and $C_{2}$ appearing in conditions $\left(S_{1}\right)$ and $\left(S_{2}\right)$ respectively, which is easy.

Lemma 4. There exists a constant $C>0$ which depends on $\Phi$ such that:

$$
a_{\varepsilon}(\tau, \tau) \geq C \varepsilon^{2}|\tau|_{V}^{2} \quad \forall \tau \in K(\Phi) .
$$

Lemma 5. There exists a constant $C>0$ independent of $\varepsilon$ such that:

$$
\sup _{\tau \in V} \frac{b(\tau, q)}{\|\tau\|_{V}} \geq C \frac{1}{\varepsilon}\|q\|_{\Sigma} \quad \forall q \in \Sigma .
$$

\section{The Discrete PROBlem}

Let us first fix notation and finite-dimensional spaces that shall be used throughout: consider $T_{h}$ a regular partition (see Ciarlet [13]) of the closed interval $I=[0,1]$ into elements $K_{i}=\left[x_{i}, x_{i+1}\right] ; i=0, N$ such that the mesh parameter, $h=\max _{1 \leq i \leq N+1} h_{i}$, with $h_{i}=\left(x_{i+1}-x_{i}\right)$ and $N \geq 1$ an integer. Let $P_{r}\left(K_{i}\right)$ be the space of polynomials of degree less or equal to $r$ defined on an element $K_{i}$. Designate by $u_{h}=\left(\beta_{h}, \gamma_{h}, \theta_{h}, \eta_{h}\right)$ the primal discrete unknown and by $v_{h}=\left(\underline{\beta}_{h}, \underline{\gamma}_{h}, \underline{\theta}_{h}, \underline{\eta}_{h}\right)$ the corresponding variable. Denote by $p_{h}=\left(p_{1 h}, p_{2 h}\right)$ the unknown in the Lagrange multipliers space and by $q_{h}=\left(q_{1 h}, q_{2 h}\right)$ the associated variable. Let $M_{h}^{l}(I)$ be the space of $C^{-1}$ piecewise polynomial interpolations of degree $l$, i.e.

$$
M_{h}^{l}=\left\{\lambda_{h} \in L^{2}(I),\left.\lambda_{h}\right|_{K_{i}} \in P_{l}(K i), i=0, N\right\} .
$$

Let $W_{h}^{k}(I)$ be the space of $C^{0}$ piecewise polynomial interpolations of degree $k$ with zero value at $x=0$ and $x=1$, i.e.

$$
W_{h}^{k}=\left\{\mu_{h} \in C(I),\left.\mu_{h}\right|_{K_{i}} \in P_{k}(K i), i=0, N \text { and } \mu_{h}(0)=\mu_{h}(1)=0\right\} .
$$

Consider, for $k, l \geq 1$, the finite-dimensional spaces:

$$
\begin{aligned}
V_{h} & =V_{h}^{k}=W_{h}^{k} \times W_{h}^{k} \times W_{h}^{k} \times M_{h}^{k-1} \\
\Sigma_{h} & =\Sigma_{h}^{l}=M_{h}^{l} \times M_{h}^{l}
\end{aligned}
$$

as the discrete spaces of primal and Lagrange multipliers variables respectively. 


\subsection{The discrete formulation}

The method consists to approach the shape function $\Phi$ by its interpolate function $\Phi_{h}$ on the space $W_{h}^{m}$, $(m \leq s)$, and set the discrete problem $\left(P_{h}\right)$ as follows: find $w_{h} \in V_{h}$ and $p_{h} \in \Sigma_{h}$ such that:

$$
\begin{aligned}
a_{h}\left(w_{h}, \tau_{h}\right)+b_{h}\left(\tau_{h}, p_{h}\right) & =l_{h}\left(\tau_{h}\right) & & \forall \tau \in V_{h} \\
b_{h}\left(w_{h}, q_{h}\right) & =0 & & \forall q_{h} \in \Sigma_{h} .
\end{aligned}
$$

Where:

$$
\begin{aligned}
a_{h}\left(w_{h}, \tau_{h}\right) & =\int_{0}^{1} \eta_{h} \underline{\eta}_{h} S\left(\Phi_{h}\right) \mathrm{d} t+\frac{\varepsilon^{2}}{12} \int_{0}^{1} \theta_{h}^{\prime} \underline{\theta}_{h}^{\prime} \cdot \frac{1}{S\left(\Phi_{h}\right)} \mathrm{d} t \\
b_{h}\left(\tau_{h}, q_{h}\right) & =\frac{1}{\varepsilon E}\left(\underline{\beta}_{h}^{\prime}-\underline{\theta}_{h} \Phi_{h}^{\prime}-\underline{\eta}_{h}, q_{1 h}\right)+\frac{1}{\varepsilon E}\left(\underline{\gamma}_{h}^{\prime}+\underline{\theta}_{h}-\Phi_{h}^{\prime} \underline{\eta}_{h}, q_{2 h}\right)
\end{aligned}
$$

and

$$
l_{h}\left(\tau_{h}\right)=-\rho\left(\underline{\gamma}_{h}, S\left(\Phi_{h}\right)\right)_{0, I}
$$

These approximations are conforming since we have:

$$
W_{h}^{m} \subset W^{1, \infty}(I), V_{h} \subset V \text { and } \Sigma_{h} \subset \Sigma
$$

We first recall from [18] the following technical result:

Lemma 6. Assume that $\Phi_{h}$ is an interpolant of $\Phi \in \Lambda_{s}$ on the space $W_{h}^{m},(m \leq s)$, then the subspace: $K_{h}\left(\Phi_{h}\right)=\left\{v_{h}=\left(\underline{\beta}_{h}, \underline{\gamma}_{h}, \underline{\theta}_{h}, \underline{\eta}_{h}\right) \in V_{h} / b_{h}\left(v_{h}, q_{h}\right)=0 \forall q_{h} \in \sum_{h}\right\}$ is nothing but the space of functions $v_{h}$ such that:

$$
\underline{\beta}_{h}^{\prime}=\underline{\theta}_{h} \Phi_{h}^{\prime}+\underline{\eta}_{h} \text { and } \gamma_{h}^{\prime}=-\underline{\theta}_{h}+\Phi_{h}^{\prime} \underline{\eta}_{h}
$$

otherwise, one has: $K_{h}\left(\Phi_{h}\right) \subset K\left(\Phi_{h}\right)$.

Lemma 7. Under the same hypotheses as in Lemma 6, the two stability conditions also hold for the approximated bilinear forms $a\left(\Phi_{h} ; .,.\right)$. and $b\left(\Phi_{h} ; .,.\right)$, indeed there exist constants $\alpha_{1}$ and $\alpha_{2}$ independents of $h$ and $\varepsilon$ such that for $h \leq h_{0}$, we have

$$
\begin{aligned}
a_{\varepsilon}\left(\Phi_{h} ; \tau_{h}, \tau_{h}\right) & \geq \alpha_{1} \varepsilon^{2}\left\|\tau_{h}\right\|_{V}^{2} & \forall \tau_{h} \in K_{h}\left(\Phi_{h}\right) \\
\sup _{\tau_{h} \in V_{h}} \frac{b\left(\Phi_{h} ; \tau_{h}, q\right)}{\left\|\tau_{h}\right\|_{V}} & \geq \alpha_{2} \frac{1}{\varepsilon}\|q\|_{\Sigma} & \forall q_{h} \in \Sigma_{h} .
\end{aligned}
$$

Proof. It suffices to remark that $S\left(\Phi_{h}\right) \geq$ land that $\frac{1}{S\left(\Phi_{h}\right)}$ is bounded bellow for $h \leq h_{0}$ with a constant independent of $h$. The proof of estimate (22) is then quite similar to this of estimate (15) in Lemma 4 . For the estimate (23), denote for any $t_{h} \in \Sigma_{h}, t_{h}^{0}$ the mean value of $t_{h}$ i.e. $t_{h}^{0}=\int_{0}^{1} t_{h} \mathrm{~d} x$. Now, for $k \geq l-1$,select, for any $q_{h} \in \Sigma_{h}, \tilde{\tau}=\left(s_{h}, t_{h}, 0,0\right)$ such that:

$$
s_{h}(x)=\int_{0}^{x}\left(q_{1 h}(\zeta)-q_{1 h}^{0}\right) \mathrm{d} \zeta \text { and } t_{h}(x)=\int_{0}^{x}\left(q_{2 h}(\zeta)-q_{2 h}^{0}\right) \mathrm{d} \zeta
$$


so that $b\left(\Phi_{h} ; \tilde{\tau}, q_{h}\right)=\left\|q_{h}\right\|_{\Sigma}^{2}$ and hence

$$
\varepsilon \sup _{\tau \in V_{h}} \frac{\left|b\left(\Phi_{h} ; \tau_{h}, q_{h}\right)\right|}{\|\tau\|_{V}} \geq \varepsilon \frac{b\left(\Phi_{h} ; \tilde{\tau}, q_{h}\right)}{\|\tau\|_{V}} \geq \frac{1}{2} \varepsilon \frac{\left\|q_{h}\right\|_{\Sigma}^{2}}{\left\|q_{h}\right\|_{\Sigma}} \geq \frac{1}{2} \varepsilon\left\|q_{h}\right\|_{\Sigma}
$$

yielding $\alpha_{2}=\frac{1}{2}$.

Now, using Lemma 7 and Brezzi's theorem (see [6]) the following result is immediate:

Theorem 8. Assume that $\Phi \in \Lambda_{s}(s \geq 1)$, then there exists a real $h_{0}>0$ such that, for $h \leq h_{0}$, the discrete problem $\left(P_{h}\right)$ admits unique solution $\left(w_{h}, p_{h}\right)$.

Let us now establish some consistency error estimates:

\subsection{Consistency error estimates}

Assume that $\Phi \in \Lambda_{s}$. Let $\Phi_{h}$ be the interpolate function of $\Phi$ on the space $W_{h}^{m}(I),(m \leq s)$, such that the interpolation error satisfies:

$$
\left|\Phi-\Phi_{h}\right|_{1} \leq C h^{m}|\Phi|_{m+1}
$$

then, with $C$ a generic constant independent of $\varepsilon$ and $h$, the following results hold:

Lemma 9. There exists a real $h_{0}^{1}>0$ such that, for $h \leq h_{0}^{1}$, the bilinear form $a_{h}(\cdot, \cdot)$ is well defined on $V \times V$, moreover one has:

$$
\left|a_{\varepsilon}\left(w_{h}, \tau_{h}\right)-a_{h}\left(w_{h}, \tau_{h}\right)\right| \leq C h^{m}\left\|w_{h}\right\|_{V}\left\|\tau_{h}\right\|_{V} \quad \forall\left(w_{h}, \tau_{h}\right) \in V_{h} \times V_{h} .
$$

Proof. On each $K_{i}$ we have:

$$
\left|a_{\varepsilon}\left(w_{h}, \tau_{h}\right)-a_{h}\left(w_{h}, \tau_{h}\right)\right| \leq \int_{K i}\left|\eta_{h} \underline{\eta_{h}}\right|\left|S(\Phi)-S\left(\Phi_{h}\right)\right| \mathrm{d} t+\frac{\varepsilon^{2}}{12} \int_{K i}\left|\theta_{h}^{\prime} \underline{\theta}_{h}^{\prime}\right|\left|\frac{1}{S(\Phi)}-\frac{1}{S\left(\Phi_{h}\right)}\right| \mathrm{d} t
$$

we will give estimate for each term of the second member of (23). Since the function $x \rightarrow \sqrt{1+x^{2}}$ is globally Lipschitz on $I R$ with constant 1 we have:

$$
\left|S(\Phi)-S\left(\Phi_{h}\right)\right| \leq\left|\Phi^{\prime}-\Phi_{h}^{\prime}\right|
$$

and

$$
\left|\frac{1}{S(\Phi)}-\frac{1}{S\left(\Phi_{1}\right)}\right| \leq\left|S(\Phi)-S\left(\Phi_{h}\right)\right| \leq\left|\Phi^{\prime}-\Phi_{h}^{\prime}\right| .
$$

By using the Schwarz inequality we get for the first term:

$$
\int_{K i}\left|\eta_{h} \underline{\eta}_{h}\right|\left|S(\Phi)-S\left(\Phi_{h}\right)\right| \mathrm{d} t \leq\left|\Phi^{\prime}-\Phi_{h}^{\prime}\right|_{0, K i}\left\|\eta_{h}\right\|_{0, K i}\left\|\underline{\eta_{h}}\right\|_{0, K i} .
$$

Now, from the interpolation error we get,with $C$ a constant independent of $h$ :

$$
\int_{K i}\left|\eta_{h} \underline{\eta}_{h}\right|\left|S(\Phi)-S\left(\Phi_{h}\right)\right| \mathrm{d} t \leq c h^{m}\left\|\eta_{h}\right\|_{0, K i} \cdot\left\|\underline{\eta}_{h}\right\|_{0, K i} .
$$


Similarly, for the second term we obtain :

$$
\frac{\varepsilon^{2}}{12} \int_{K i}\left|\theta_{h}^{\prime} \underline{\theta}_{h}^{\prime}\right|\left|\frac{1}{S(\Phi)}-\frac{1}{S(\Phi)_{h}}\right| \mathrm{d} t \leq \frac{\varepsilon^{2}}{12}\left|\theta_{h}\right|_{1, K i}\left|\underline{\theta}_{h}\right|_{1, K i}\left|\Phi-\Phi_{h}\right|_{0, K i} .
$$

So, from (24) we get:

$$
\frac{\varepsilon^{2}}{12} \int_{K i}\left|\theta_{h}^{\prime} \underline{\theta}_{h}^{\prime}\right|\left|\frac{1}{S(\Phi)}-\frac{1}{S(\Phi)_{h}}\right| \mathrm{d} t \leq \operatorname{ch}^{m} \frac{\varepsilon^{2}}{12}\left|\theta_{h}\right|_{1, K i}\left|\underline{\theta}_{h}\right|_{1, K i}
$$

Adding and using the Schwarz inequality, we obtain the estimate after summing the integrals over all elements $K_{i}$.

Lemma 10. There exists a real $h_{0}^{2}>0$ such that, for $h \leq h_{0}^{2}$, the bilinear form $b_{h}(\cdot, \cdot)$ on $V \times \Sigma$ is well defined and:

$$
\left|b_{h}\left(\tau_{h}, q_{h}\right)-b\left(\tau_{h}, q_{h}\right)\right| \leq C \frac{h^{m}}{\varepsilon}\left\|\tau_{h}\right\|_{V}\left\|q_{h}\right\|_{\Sigma} \forall \tau \in V_{h} ; \quad \forall q \in \Sigma_{h}
$$

Proof. For any $\tau_{h}=\left(\beta_{h}, \gamma_{h}, \theta_{h}, \eta_{h}\right) \in V_{h}$ and any $q_{h}=\left(q_{1 h}, q_{2 h}\right) \in \Sigma_{h}$ we have:

$$
\left|b\left(\tau_{h}, q_{h}\right)-b_{h}\left(\tau_{h}, q_{h}\right)\right| \leq \frac{C}{\varepsilon} \int_{0}^{1}\left(\left|\theta_{h} q_{1 h}\right|\left|\Phi^{\prime}-\Phi_{h}^{\prime}\right|+\left|\eta_{h} q_{2 h}\right|\left|\Phi^{\prime}-\Phi_{h}^{\prime}\right|\right) \mathrm{d} t .
$$

From (24) and the Schwarz inequality yields:

$$
\left|b\left(\tau_{h}, q_{h}\right)-b_{h}\left(\tau_{h}, q_{h}\right)\right| \leq C \frac{h^{m}}{\varepsilon}|\Phi|_{m+1, K}\left(\left\|\theta_{h}\right\|_{0, K}\left\|q_{1 h}\right\|_{0, K}+\left\|\eta_{h}\right\|_{0, K}\left\|q_{2 h}\right\|_{0, K}\right) .
$$

Thus, with $C$ a constant which depends on $\Phi$ and doesn't depend on $\varepsilon$ and $h$ :

$$
\left|b\left(\tau_{h}, q_{h}\right)-b_{h}\left(\tau_{h}, q_{h}\right)\right| \leq C \frac{h^{m}}{\varepsilon}\left(\left\|\theta_{h}\right\|_{0}+\left\|\eta_{h}\right\|_{0}\right)\left(\left\|q_{1 h}\right\|_{0}+\left\|q_{2 h}\right\|_{0}\right)
$$

Lemma 11. There exists a real $h_{0}^{3}>0$ such that, for $h \leq h_{0}^{3}$, the linear form $l_{h}(\cdot)$ on $V_{h}$ is well defined and

$$
\left|l\left(\tau_{h}\right)-l_{h}\left(\tau_{h}\right)\right| \leq c h^{m}\left\|\tau_{h}\right\|_{V} \quad \forall \tau_{h} \in V_{h}
$$

Proof. For any $\tau_{h}=\left(\beta_{h}, \gamma_{h}, \theta_{h}, \eta_{h}\right) \in V_{h}$ we have:

$$
\left|l\left(\tau_{h}\right)-l_{h}\left(\tau_{h}\right)\right| \leq \rho\left|\int_{0}^{1} \gamma\left(S(\Phi)-S\left(\Phi_{h}\right)\right) \mathrm{d} t\right|
$$

Using the Schwarz inequality and (24) we immediately obtain

$$
\left|l\left(\tau_{h}\right)-l_{h}\left(\tau_{h}\right)\right| \leq C h^{m}|\Phi|_{m+1}\|\gamma\|_{0} .
$$

\subsection{Accuracy error estimate}

We can now prove the error estimates theorem. Designating by $C$ a generic constant independent of $\varepsilon$ and $h$, the following holds: 
Theorem 12. Let, for $\Phi \in \Lambda_{s}(s \geq 1),(u, p)$ be the solution of problem $\left(P_{\varepsilon}\right)$ and $\left(u_{h}, p_{h}\right)$ be the solution of problem $\left(P_{h}\right)$ then we have:

$$
\begin{aligned}
\left\|u-u_{h}\right\|_{V} \leq \frac{C}{\varepsilon^{2}}\left\{\inf _{\tau_{h} \in V_{h}}\left\|u-\tau_{h}\right\|_{V}+\inf _{\tau_{h} \in V_{h}} \sup _{v_{h} \in V_{h}} \frac{\left|a_{h}\left(\tau_{h}, v_{h}\right)-a\left(\tau_{h}, v_{h}\right)\right|}{\left\|v_{h}\right\|_{V}}\right. \\
\left.+\sup _{v_{h} \in V_{h}} \frac{\left|b_{h}\left(v_{h}, p\right)-b\left(v_{h}, p\right)\right|}{\left\|v_{h}\right\|_{V}}+\sup _{v_{h} \in V_{h}} \frac{\left|l\left(v_{h}\right)-l_{h}\left(v_{h}\right)\right|}{\left\|v_{h}\right\|_{V}}\right\} .
\end{aligned}
$$

Assume that, $u \in\left\{H^{k+1}(I) \cap H_{0}^{1}(I)\right\}^{3} \times H^{k}(I)$ and $p \in\left\{H^{l+1}(I) \times H^{l+1}(I)\right\}$. Let $\tilde{u}_{h} \in V_{h}, \tilde{p}_{h} \in \sum_{h}$ and $\Phi_{h} \in W_{h}$ be interpolants of $u, p$ and $\Phi$ respectively. Assume that the interpolation errors $u-\tilde{u}_{h}, p-\tilde{p}_{h}$ and $\Phi-\Phi_{h}$ satisfy

$$
\begin{aligned}
\left\|u-\tilde{u}_{h}\right\|_{V} & \leq C h^{k} \\
\left\|p-\tilde{p}_{h}\right\|_{\sum} & \leq C h^{l+1} \\
\left\|\Phi-\Phi_{h}\right\|_{1} & \leq C h^{m}
\end{aligned}
$$

then, with $\nu=\min (k, l+1, m)$ :

$$
\left\|u-u_{h}\right\|_{V} \leq C(p, u) \varepsilon^{-2} h^{\nu} .
$$

In particular for $m=1$ (when the arch is approximated by straight beams) we have, for any higher order elements

$$
\left\|u-u_{h}\right\|_{V} \leq C(p, u) h \varepsilon^{-2}
$$

and hence a locking phenomenon could appear when $(\varepsilon \prec \sqrt{h})$.

Proof. As the problem $\left(P_{h}\right)$ satisfies the general hypotheses of Brezzi's theorem, the technique used to prove estimate (29) is classical (see [8], p. 67). For the usual term $\inf _{\tau_{h} \in V_{h}}\left\|u-\tau_{h}\right\|_{V}$ of the right hand side of inequality (30) the infinimum is bounded from above taking $\tau_{h}=\tilde{\tau}_{h}$ the $V_{h}$-interpolate of $\tau$. The additional tree terms which measure the consistency between the original forms and their approximated ones, are given by Lemmas 7 to 9 . Indeed from Lemma 7 we have

$$
\left|a\left(\tilde{u}_{h}, v_{h}\right)-a_{h}\left(\tilde{u}_{h}, v_{h}\right)\right| \leq C h^{m}\left\|\tilde{u}_{h}\right\|_{V}\left\|v_{h}\right\|_{V}
$$

so that

$$
\sup _{v_{h} \in V_{h}} \frac{\left|a\left(\tilde{u}_{h}, v_{h}\right)-a_{h}\left(\tilde{u}_{h}, v_{h}\right)\right|}{\left\|v_{h}\right\|_{V}} \leq C h^{m}\left\|\tilde{u}_{h}\right\|_{V} .
$$

Similarly, by Lemma 8 we have, with $\tilde{q}_{h}$ the $\Sigma_{h}$-interpolate of $q$

$$
\sup _{v_{h} \in V_{h}} \frac{\left|b_{h}\left(v_{h}, \tilde{q}_{h}\right)-b\left(v_{h}, \tilde{q}_{h}\right)\right|}{\left\|v_{h}\right\|_{V}} \leq C \frac{h^{m}}{\varepsilon}\left\|\tilde{q}_{h}\right\|_{\Sigma} .
$$

Since the operator of $V_{h}$-interpolation (resp. $\Sigma_{h}$-interpolation) satisfy (see [8])

$$
\left\|\tilde{u}_{h}\right\|_{V} \leq C\|u\|_{V} \text { and }\left\|\tilde{q}_{h}\right\|_{\Sigma} \leq C\|q\|_{\Sigma}
$$

the error estimate (33) follows by combining (28) to (32) and (33) to (36). 


\section{The Residual-Free BubBles technique}

It was established that standard Galerkin method do not be effective to give a suitably approximation of the solution of problem $\left(P_{\varepsilon}\right)$ when $\varepsilon \ll h$. In order to achieve good approximation properties, we propose to enrich the Galerkin method by residual-free bubbles functions [22]. To be more precise, for the given grid $T_{h}$, we still employ piecewise linear elements to approach the geometry of the arch, but for approximation of the displacement, we add to the space $V_{h}$ of polynomials a space spanned by bubbles functions to recover an augmented test space $S_{h}$. The bubble functions are assumed to satisfy the governing differential equations in each element interior subjected to the Dirichlet conditions on each element boundary. Denote:

$$
\begin{aligned}
V_{l} & =W_{h}^{1} \times W_{h}^{1} \times W_{h}^{1} \times W_{h}^{1} \\
\Sigma_{l} & =\Sigma_{h}^{1}
\end{aligned}
$$

where $W_{h}^{1}$ and $\Sigma_{l}^{1}$ are defined as in Section 3. Let $V_{b}$ and $\Sigma_{b}$ designate the spaces spanned by bubbles corresponding to $V_{l}$ and $\Sigma_{l}$ respectively. The new test spaces are

$$
S_{h}=V_{l} \oplus V_{b} \quad \text { and } \quad \Sigma_{h}=\Sigma_{l} \oplus \Sigma_{b}
$$

Consequently each $w_{h} \in S_{h}$ and each $p_{h}$ of $\Sigma_{h}$ is the sum of a polynomial and a bubble component:

$$
w_{h}=w_{l}+w_{b} ; p_{h}=p_{l}+p_{b} .
$$

Let us now shed some light on the effect of eliminating the bubble functions by the static condensation procedure. For simplicity writing, we shall drop the subscript $\varepsilon$ in the forms $a_{\varepsilon h}(.,$.$) and l_{\varepsilon h}($.$) . Consider the problem:$ find $w_{h} \in S_{h}$ and $p_{h} \in \Sigma_{h}$ such that:

$$
\left(P_{h}\right): \begin{cases}a_{h}\left(w_{h}, \tau_{h}\right)+b_{h}\left(\tau_{h}, p_{h}\right)=l_{h}\left(\tau_{h}\right) & \forall \tau_{h} \in S_{h} \\ b_{h}\left(w_{h}, q_{h}\right)=0 & \forall q_{h} \in \Sigma_{h}\end{cases}
$$

where, with $w_{l}=\left(\beta_{l}, \gamma_{l}, \theta_{l}, \eta_{l}\right) ; \tau_{l}=\left(\underline{\beta}_{l}, \underline{\gamma}_{l}, \underline{\theta}_{l}, \underline{\eta}_{l}\right) ; w_{b}=\left(\beta_{b}, \gamma_{b}, \theta_{b}, \eta_{b}\right)$ and $\tau_{b}=\left(\underline{\beta}_{b}, \underline{\gamma}_{b}, \underline{\theta}_{b}, \underline{\eta}_{b}\right)$

$$
\begin{aligned}
a_{h}\left(w_{h}, \tau_{h}\right) & =\varepsilon \int_{0}^{1} \eta_{h} \underline{\eta}_{h} S\left(\Phi_{h}\right) \mathrm{d} t+\frac{\varepsilon^{3}}{12} \int_{0}^{1} \theta_{h}^{\prime} \underline{\theta}_{h}^{\prime} \frac{1}{S\left(\Phi_{h}\right)} \mathrm{d} t \\
b_{h}\left(\tau_{h}, q_{h}\right) & =\left(\underline{\beta}_{h}^{\prime}-\underline{\theta}_{h} \Phi_{h}^{\prime}-\underline{\eta}_{h}, q_{1 h}\right)+\left(\gamma_{h}^{\prime}+\underline{\theta}_{h}-\Phi_{h}^{\prime} \underline{\eta}_{h}, q_{2, h}\right) \\
l_{h}\left(\tau_{h}\right) & =-\varepsilon \int_{0}^{1} \underline{\gamma}_{h} S\left(\Phi_{h}\right) \mathrm{d} t .
\end{aligned}
$$

Reporting in $\left(P_{h}\right)$ yields:

$$
\begin{aligned}
& a_{h}\left(w_{l}+w_{b}, \tau_{l}+\tau_{b}\right)+b_{h}\left(\tau_{l}+\tau_{b}, p_{l}+p_{b}\right)=l_{h}\left(\tau_{l}+\tau_{b}\right) \quad \forall \tau_{l} \in V_{l} \quad \forall \tau_{b} \in V_{b} \\
& b_{h}\left(w_{l}+w_{b}, q_{l}+q_{b}\right)=0 \quad \forall q_{l} \in \Sigma_{l} \quad \forall q_{b} \in \Sigma_{b} .
\end{aligned}
$$

Now, if we take first $\tau_{l}=0$ and $q_{l}=0$ we get the formulation:

$$
\begin{aligned}
a_{h}\left(w_{b}, \tau_{b}\right)+b_{h}\left(\tau_{b}, p_{b}\right) & =l_{h}\left(\tau_{b}\right)-a_{h}\left(w_{l}, \tau_{b}\right)-b_{h}\left(\tau_{b}, p_{l}\right) & & \forall \tau_{b} \in V_{b} \\
b_{h}\left(w_{b}, q_{b}\right) & =-b_{h}\left(w_{l}, q_{b}\right) & & \forall q_{b} \in \Sigma_{b} .
\end{aligned}
$$


Equivalently, with $A$ and $B$ the operators associated to the forms $a_{\varepsilon}(.,$.$) and b(.,$.

$$
\begin{aligned}
a_{h}\left(w_{b}, \tau_{b}\right)+b_{h}\left(\tau_{b}, p_{b}\right) & =\left\langle f-A w_{l}-B^{*} p_{l}, \tau_{b}\right\rangle & & \forall \tau_{b} \in V_{b} \\
b_{h}\left(w_{b}, q_{b}\right) & =-\left\langle B w_{l}, q_{b}\right\rangle & & \forall q_{b} \in \sum_{b} .
\end{aligned}
$$

Consequently, for the given $\left(w_{l}, p_{l}\right) \in V_{l} \times \Sigma_{l}$, the pair of bubbles $\left(w_{b}, p_{b}\right)$ satisfy:

$$
\begin{aligned}
A w_{b}+B^{*} p_{b} & =-A w_{l}-B^{*} p_{l}-f & & \text { in } K \\
B w_{b} & =-B w_{l} & & \text { in } K \\
w_{b} & =p_{b}=0 & & \text { on } \partial K .
\end{aligned}
$$

Selecting $\tau_{b}=q_{b}=0$ in (34) yields:

$$
\begin{aligned}
a_{h}\left(w_{b}+w_{l}, \tau_{l}\right)+b_{h}\left(\tau_{l}, p_{l}+p_{b}\right) & =l_{h}\left(\tau_{l}\right) & & \forall \tau_{l} \in V_{l} \\
b_{h}\left(w_{b}+w_{l}, q_{l}\right) & =0 & & \forall q_{l} \in \sum_{l} .
\end{aligned}
$$

Which can be written as:

$$
\begin{aligned}
& a_{h}\left(w_{l}, \tau_{l}\right)+\left\langle A w_{b}+B^{*} p_{b}, \tau_{l}\right\rangle+b_{h}\left(\tau_{l}, p_{l}\right)=l_{h}\left(\tau_{l}\right) \quad \forall \tau_{l} \in V_{l} \\
& b_{h}\left(w_{l}, q_{l}\right)=-b_{h}\left(w_{b}, q_{l}\right) \quad \forall q_{l} \in \sum_{l} .
\end{aligned}
$$

Where the term $\left\langle A w_{b}+B^{\star} p_{b}, \tau_{l}\right\rangle$ gives the bubbles contribution to the reduced space formulation.

Determination of the bubble basis functions. Let, for $i=1, n_{K},\left\{\Psi_{l i}\right\}$ and $\left\{p_{l i}\right\}$ be the polynomial local basis corresponding to $V_{l}$ and $\sum_{l}$ respectively; $\left\{\Psi_{b_{i}}, \Psi_{f}\right\}$ and $\left\{p_{b i}, p_{f}\right\}$ denote the local basis of bubble functions associated to $V_{b}$ and $\sum_{b}$. Problem (36) splits in: find $\Psi_{b i}$ and $p_{b i}$ such that

$$
\begin{aligned}
& A \Psi_{b i}+B^{*} p_{b i}=-A \Psi_{l i}+B^{*} p_{l i} \quad i=1, n_{K} \quad \text { in } K \\
& B \Psi_{b i}=-B \Psi_{l i} \quad i=1, n_{K} \quad \text { in } K \\
& \Psi_{b i}=p_{b i}=0 \quad i=1, n_{K} \quad \text { on } \partial K
\end{aligned}
$$

and, find $\Psi_{b f}$ and $p_{f}$ such that

$$
\begin{aligned}
A \Psi_{b f} & =-B^{*} p_{l}-f & & \text { in } K \\
B \Psi_{b f} & =0 & & \text { in } K \\
\Psi_{b f} & =0 & & \text { on } \partial K .
\end{aligned}
$$

Since $\Phi_{h}$ is linear in each element $K$, for the sake of clarity, we set $\Phi_{h}^{\prime}=a_{K}=a, S\left(\Phi_{h}\right)=\sqrt{1+a_{k}^{2}}=S_{K}=S$ and $h_{K}=h$. Now, problems (38) and (39) can be solved for each $\Psi_{l i}$ and each $p_{l i}$, we have: 
Proposition 13. Assume that $\Phi \in \Lambda_{s}$ and problem $\left(P_{\varepsilon}\right)$ admits a solution $(w, p)$ such that $w \in\left(H_{0}^{1}(I) \cap H^{2}(I)\right)^{3}$ $\times\left(H^{2}(I)\right)$ and $p \in\left(H^{2}(I)\right)^{2}$ then problem (39) admits the solution $\Psi_{b f}=\left(\beta_{b f}, \gamma_{b f}, \theta_{b f}, \eta_{b f}\right)$ and $p_{f}=$ $\left(p_{f 1}, p_{f 2}\right)$ such that:

$$
\left\{\begin{array}{l}
\beta_{b f}(\xi)=-\frac{a\left(1+a^{2}\right)}{6 \varepsilon^{2}}\left[\left(\frac{\xi^{4}}{4}-\frac{\xi^{2}}{2} h^{2}\right)+\frac{1}{4} h \xi^{2}(3 h-2 \xi)\right]-a \frac{\xi^{2}}{2}+\frac{a}{2} h \xi \\
\gamma_{b f}(\xi)=\frac{a^{2}}{2} \xi(h-\xi)+\frac{1+a^{2}}{24 \varepsilon^{2}} \xi^{2}(h-\xi)^{2} \\
\theta_{b f}(\xi)=-\frac{\left(1+a^{2}\right)}{2 \varepsilon^{2}}\left[\frac{1}{3} \xi\left(\xi^{2}-h^{2}\right)+\frac{1}{2} h^{2} \xi(h-\xi)\right] \\
\left.\eta_{b f}(\xi)=a\left(\frac{h}{2}-\xi\right), \quad \xi \in\right] 0, h\left[\text { and } \eta_{b f}(0)=\eta_{b f}(1)=0\right. \\
\left\{\begin{array}{l}
p_{f 1}(\xi)=0 \\
p_{f 2}(\xi)=-\varepsilon S \xi+\frac{S}{2} h \varepsilon .
\end{array}\right.
\end{array}\right.
$$

Proof. $\Psi_{b}^{i}=\left(\beta_{b}^{i}, \gamma_{b}^{i}, \theta_{b}^{i}, \eta_{b}^{i}\right) ; \Psi_{b f}=\left(\beta_{b f}, \gamma_{b f}, \theta_{b f}, \eta_{b f}\right) ; p_{f}=\left(p_{f 1}, p_{f 2}\right) ; p_{l i}=\left(p_{l 1}^{i}, p_{l 2}^{i}\right)$.

Now, we write (with the same coefficients $C_{i, K}$ ):

$$
w_{l \mid K}=\Sigma_{i=1}^{n K} C_{i, K} \Psi_{l i} \quad \text { and } \quad w_{b \mid K}=\Sigma_{i=1}^{n K} C_{i, K} \Psi_{b}^{i}+\Psi_{b f}
$$

Hence, equations (39) can be written as:

$$
\begin{cases}(a) & p_{f 1}^{\prime}=0 \\ (b) & p_{f 2}^{\prime}=-\varepsilon S\left(\Phi_{h}\right) \\ (c) & -\varepsilon^{3} \frac{\theta_{b f}^{\prime \prime}}{S\left(\Phi_{h}\right)}=p_{f 1} \Phi_{h}^{\prime}-p_{f 2} \\ (d) & \varepsilon S\left(\Phi_{h}\right) \eta_{b f}=p_{f 1}+\Phi_{h}^{\prime} p_{f 2} \\ (e) & \beta_{b f}^{\prime}=\theta_{b f} \Phi_{h}^{\prime}+\eta_{b f} \\ (f) & \gamma_{b f}^{\prime}=\Phi_{h}^{\prime} \eta_{b f}-\theta_{b f} .\end{cases}
$$

In $(41)$, from equations $(b)$ and $(c)$ we get, respectively, after integrating once with respect to the local variable $\xi=x-x_{i} \in[0, h]$,

$$
\begin{aligned}
& p_{f 2}(\xi)=-\varepsilon S \xi+C_{1} \\
& \theta_{b f}^{\prime \prime}(\xi)=-\frac{S}{\varepsilon^{3}}\left(p_{f 1} a+\varepsilon S \xi-C_{1}\right) .
\end{aligned}
$$

Integrating twice with respect to $\xi$ and using the conditions $\theta_{b f}(0)=\theta_{b f}(h)=0$ yields

$$
\theta_{b f}(\xi)=-\frac{S^{2}}{6 \varepsilon^{2}} \xi\left(\xi^{2}-h^{2}\right)+\frac{S\left(C_{1}-p_{f 1} a\right)}{2 \varepsilon^{3}} \xi(\xi-h) .
$$

Using from (41) equation $(d)$ we obtain for $\eta_{b f}$ :

$$
\eta_{b f}=\frac{1}{\varepsilon S}\left(p_{f 1}+a p_{f 2}\right) .
$$

Hence from (42), we get:

$$
\eta_{b f}(\xi)=\frac{1}{\varepsilon S}\left(p_{f 1}+a C_{1}-a \varepsilon S \xi\right)
$$


Similarly, we use from (41) equations $(e)$ and $(f)$, to have:

$$
\beta_{b f}(\xi)=a \int_{0}^{\xi} \theta_{b f}(t) \mathrm{d} t+\int_{0}^{\xi} \eta_{b f}(t) \mathrm{d} t .
$$

Reporting expressions of $\theta_{b f}$ and $\eta_{b f}$ yields, with $d=S\left(C_{1}-p_{f 1} a\right) / \varepsilon^{3}$ and $f=-S^{2} / \varepsilon^{2}$ :

$$
\beta_{b f}(\xi)=\frac{f}{6} a\left(\frac{\xi^{4}}{4}-\frac{\xi^{2}}{2} h^{2}\right)-a \frac{d}{12} \xi^{2}(3 h-2 \xi)+\frac{1}{\varepsilon S}\left(p_{f 1}+a C_{1}\right) \xi-a \frac{\xi^{2}}{2} .
$$

Now, using the condition $\beta_{b f}(h)=0$ and replacing $f$ by its expression, we get with $\alpha=h / \varepsilon$ :

$$
C_{1}=\frac{\frac{S^{2}}{2} h^{2} \alpha^{2}+a S p_{f 1} \alpha^{3}-6 h^{2}}{S \alpha^{3}-\frac{12}{S} \alpha}+\frac{12 \frac{p_{f 1}}{S} \alpha}{a\left(S \alpha^{3}-\frac{12}{S} \alpha\right)} .
$$

Otherwise, using from (41) the equation $(f)$ and integrating once with respect to $\xi$ yields

$$
\gamma_{b f}(\xi)=a \int_{0}^{\xi} \eta_{b f}(t) \mathrm{d} t+\int_{0}^{\xi} \theta_{b f}(t) \mathrm{d} t .
$$

So, substituting with the $\eta_{b f}$ and $\theta_{b f}$ expressions we get:

$$
\gamma_{b f}(\xi)=\frac{a}{\varepsilon S}\left(p_{f 1}+a c_{1}\right) \xi-a^{2} \frac{\xi^{2}}{2}+\frac{f}{6}\left(\frac{\xi^{2}}{2} h^{2}-\frac{\xi^{4}}{4}\right)+\frac{S\left(C_{1}-p_{f 1} a\right)}{12 \varepsilon^{3}} \xi^{2}(3 h-2 \xi) .
$$

Hence, using (46) and (47) we obtain after brief calculation

$$
C_{1}=\frac{\sqrt{1+a^{2}}}{2} h \varepsilon ; \quad p_{f 1}=0 .
$$

Now, recalling (48) and reporting in (43) and (44) we obtain:

$$
\theta_{b f}(\xi)=-\frac{\left(1+a^{2}\right)}{2 \varepsilon^{2}}\left[\frac{1}{3} \xi\left(\xi^{2}-h^{2}\right)+\frac{1}{2} h^{2} \xi(h-\xi)\right]
$$

and

$$
\left\{\begin{array}{l}
\left.\eta_{b f}(\xi)=a\left(\frac{h}{2}-\xi\right), \quad \xi \in\right] 0, h[ \\
\eta_{b f}(0)=\eta_{b f}(h)=0
\end{array}\right.
$$

Similarly, replacing in (45) and (47) $C_{1}$ and $p_{f 1}$ by their expressions, we get

$$
\beta_{b f}(\xi)=-\frac{a\left(1+a^{2}\right)}{24 \varepsilon^{2}} \xi^{2}(\xi-h)^{2}+\frac{a}{2} \xi(h-\xi)
$$

and

$$
\gamma_{b f}(\xi)=\frac{a^{2}}{2} \xi(h-\xi)+\frac{1+a^{2}}{24 \varepsilon^{2}} \xi^{2}(h-\xi)^{2} .
$$


Now, it remains to determine the local basis elements $\Psi_{b}^{i}$ and $p_{b}^{i}$. Let us, for the sake of clarity, introduce the quantities:

$$
\begin{cases}m_{1}^{1}=\frac{12(a-1)}{S^{3}} \frac{\varepsilon^{3}}{h^{3}}-\frac{6}{S} \frac{\varepsilon^{3}}{h^{2}} & m_{1}^{2}=-\frac{6}{S}\left(\frac{4}{a}+h\right) \frac{\varepsilon^{3}}{h^{3}} \\ m_{2}^{1}=\frac{a+1}{S} \frac{\varepsilon}{h} & m_{2}^{2}=S \frac{\varepsilon}{h}\end{cases}
$$

With the notation above, the following holds:

Proposition 14. Under the same hypotheses as in proposition 15, problem (39) admits the solutions $\Psi_{b}^{i}=$ $\left(\beta_{b}^{i}, \gamma_{b}^{i}, \theta_{b}^{i}, \eta_{b}^{i}\right)$ and $p_{b}^{i}=\left(p_{b, 1}^{i}, p_{b, 2}^{i}\right) ; i=1,2$ such that

$$
\begin{aligned}
& (a)\left\{\begin{array}{l}
\beta_{b}^{1}(\xi)=-\frac{a S}{6 \varepsilon^{3}} m_{1}^{1} \xi^{3}-\left(\frac{a S m_{1}^{1} h}{4 \varepsilon^{3}}+\frac{a}{2 h}\right) \xi^{2}+\left(\frac{m_{2}^{1}}{\varepsilon S}-\frac{1}{h}\right) \xi \\
\gamma_{b}^{1}(\xi)=\frac{S}{6 \varepsilon^{3}} m_{1}^{1} \xi^{3}-\left(\frac{S m_{1}^{1} h}{4 \varepsilon^{3}}+\frac{1}{2 h}\right) \xi^{2}+\left(\frac{a m_{2}^{1}}{\varepsilon S}-\frac{1}{h}\right) \xi \\
\theta_{b}^{1}(\xi)=\frac{S m_{1}^{1}}{2 \varepsilon^{3}} \xi(h-\xi) \\
\eta_{b}^{1}(\xi)=-\frac{\xi}{h}+\frac{m_{2}^{1}}{\varepsilon S_{K}}
\end{array}\right. \\
& (b)\left\{\begin{array}{l}
p_{b 1}^{1}(\xi)=-\frac{\xi}{h}+\frac{a+1}{S^{3}} \frac{\varepsilon}{h}-\frac{6 a}{S^{3}} \frac{\varepsilon^{3}}{h^{2}}+\frac{12 a(a-1)}{S^{5}} \frac{\varepsilon^{3}}{h^{3}} \\
p_{b 2}^{1}(\xi)=-\frac{\xi}{h}-\frac{(a+1) a}{S^{3}} \frac{\varepsilon}{h}-\frac{6}{S^{3}} \frac{\varepsilon^{3}}{h^{2}}+\frac{12(a-1)}{S^{5}} \frac{\varepsilon^{3}}{h^{3}}
\end{array}\right.
\end{aligned}
$$

and

$$
\begin{aligned}
& (a)\left\{\begin{array}{l}
\beta_{b}^{2}(\xi)=\left\{-\frac{a S}{6 \varepsilon^{3}} m_{1}^{2} \xi^{3}+\left(\frac{a S m_{1}^{2} h}{4 \varepsilon^{3}}-\frac{a}{2 h}\right) \xi^{2}+\left(\frac{m_{2}^{2}}{\varepsilon S}+\frac{1}{h}+a\right) \xi\right. \\
\gamma_{b}^{2}(\xi)=\left\{\frac{S}{6 \varepsilon^{3}} m_{1}^{2} \xi^{3}-\left(\frac{S m_{1}^{2} h}{4 \varepsilon^{3}}-\frac{1}{2 h}\right) \xi^{2}+\left(\frac{a m_{2}^{2}}{\varepsilon S}+\frac{1}{h}-1\right) \xi\right. \\
\theta_{b}^{2}(\xi)=\frac{S m_{1}^{2}}{2 \varepsilon^{3}} \xi(h-\xi) \\
\eta_{b}^{2}(\xi)=-1+\frac{\xi}{h}+\frac{m_{2}^{2}}{\varepsilon S_{K}}
\end{array}\right. \\
& (b)\left\{\begin{array}{l}
p_{b 1}^{2}(\xi)=\frac{\xi}{h}-1-\frac{6}{S^{3}}(4+a h) \frac{\varepsilon^{3}}{h^{3}}+\frac{1}{S} \frac{\varepsilon}{h} \\
p_{b 2}^{2}(\xi)=\frac{\xi}{h}-1-\frac{6}{S^{3}}\left(\frac{4}{a}+h\right) \frac{\varepsilon^{3}}{h^{3}}-\frac{a}{S} \frac{\varepsilon}{h} .
\end{array}\right.
\end{aligned}
$$


Proof. Denote first, for $i=1, n_{K}, \Psi_{l}^{i}=\left(\beta_{l}^{i}, \gamma_{l}^{i}, \theta_{l}^{i}, \eta_{l}^{i}\right)$ and let us ignore the subscript i in the proof. Using in (38) equations $(a)$ and $(b)$, we get for $\left(w_{l}, p_{l}\right) \in V_{l} \times \Sigma_{l}$ :

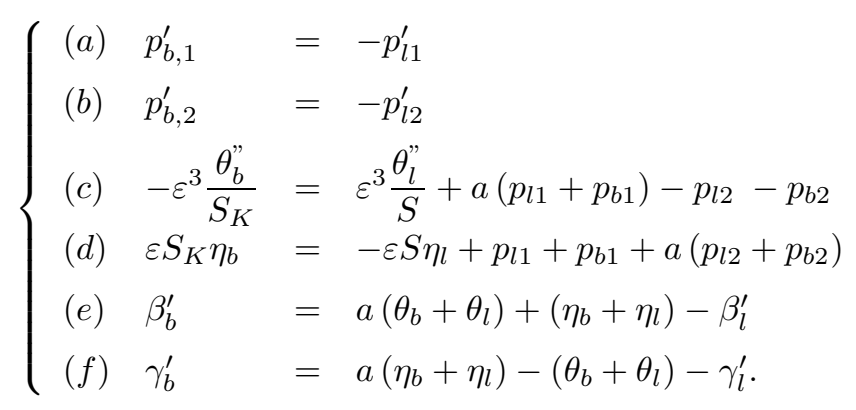

Hence, from equations (a) and (b) yields, with $M_{1}$ and $M_{2}$ generic constants:

$$
p_{b 1}=-p_{l 1}+M_{1} ; p_{b 2}=-p_{l 2}+M_{2} .
$$

Now, equation (d) gives:

$$
\eta_{b}=-\eta_{l}+\frac{1}{\varepsilon S}\left[M_{1}+a M_{2}\right]
$$

Since $\theta_{l}$ is linear in $K$, we have $\theta_{l}^{\prime \prime}=0$ and then from (c) yields:

$$
\theta_{b}^{\prime \prime}=-\frac{S}{\varepsilon^{3}}\left(a M_{1}-M_{2}\right) .
$$

As the elements $\theta_{l}, \beta_{l}, \gamma_{l}, p_{l 1}$ and $p_{l 2}$ are linears in $K$, one has:

$$
\left\{\begin{array}{l}
\text { (i) } \quad \theta_{l}=\theta_{l}^{1}=\beta_{l}=\beta_{l}^{1}=\gamma_{l}=\gamma_{l}^{1}=p_{l 1}^{1}=p_{l 2}^{1}=\frac{\xi}{h} \\
(\text { ii }) \quad \theta_{l}=\theta_{l}^{2}=\beta_{l}=\beta_{l}^{2}=\gamma_{l}=\gamma_{l}^{2}=p_{l 1}^{2}=p_{l 2}^{2}=1-\frac{\xi}{h} .
\end{array}\right.
$$

In the first case we get:

$$
p_{b 1}^{1}=-\frac{\xi}{h}+M_{1}^{1} ; p_{b 2}^{1}=-\frac{\xi}{h}+M_{2}^{1}
$$

Setting: $m_{1}^{1}=a M_{1}^{1}-M_{2}^{1}$ and $m_{2}^{1}=M_{1}^{1}+a M_{2}^{1}$ yields:

$$
\eta_{b}^{1}(\xi)=-\frac{\xi}{h}+\frac{m_{2}^{1}}{\varepsilon S}
$$

Integrating twice with respect to $\xi$ and recalling $\theta_{b}(0)=\theta_{b}(h)=0$, we obtain

$$
\theta_{b}^{1}(\xi)=\frac{S m_{1}^{1}}{2 \varepsilon^{3}} \xi(h-\xi) .
$$

Otherwise, using from (57) equation (e) and integrating, we get:

$$
\beta_{b}(\xi)=a \int_{0}^{\xi}\left(\theta_{b}+\theta_{l}\right)(t) \mathrm{d} t+\int_{0}^{\xi}\left(\eta_{b}+\eta_{l}\right)(t) \mathrm{d} t-\beta_{l}(\xi)+\beta_{l}(0)
$$


so according to (60) and (61)

$$
\beta_{b}^{1}(\xi)=-\frac{a S}{6 \varepsilon^{3}} m_{1}^{1} \xi^{3}-\left(\frac{a S m_{1}^{1} h}{4 \varepsilon^{3}}+\frac{a}{2 h}\right) \xi^{2}+\left(\frac{m_{2}^{1}}{\varepsilon S}-\frac{1}{h}\right) \xi .
$$

Similarly, using equation $(f)$ and integrating with respect to $\xi$ we obtain:

$$
\gamma_{b}(\xi)=a \int_{0}^{\zeta}\left(\eta_{b}+\eta_{l}\right)(t) \mathrm{d} t-\int_{0}^{\zeta}\left(\theta_{b}+\theta_{l}\right)(t) \mathrm{d} t-\gamma_{l}(\xi)+\gamma_{l}(0)
$$

Hence, from (60) and (61):

$$
\gamma_{b}^{1}(\xi)=\frac{S}{6 \varepsilon^{3}} m_{1}^{1} \xi^{3}-\left(\frac{S m_{1}^{1} h}{4 \varepsilon^{3}}+\frac{1}{2 h}\right) \xi^{2}+\left(\frac{a m_{2}^{1}}{\varepsilon S}-\frac{1}{h}\right) \xi .
$$

In other words:

$$
\gamma_{b}^{1}(\xi)=-\frac{\beta_{b}^{1}(\xi)}{a}+\left(-\frac{a+1}{a h}+\frac{a^{2}+1}{a S \varepsilon} m_{2}^{1}\right) \xi
$$

Finally it remains to determine the constants $m_{1}^{1}$ and $m_{2}^{1}$. This can be done by the use of the conditions $\beta_{b}^{1}(h)=\gamma_{b}^{1}(h)=0$ According to $(63)$ we have:

$$
m_{2}^{1}=\frac{a+1}{S} \frac{\varepsilon}{h}
$$

On the other hand, using the expression of $\beta_{b}^{1}$, we get after brief calculation

$$
\frac{a S}{12} m_{1}^{1} \frac{h^{2}}{\varepsilon^{3}}+\frac{1}{\varepsilon S} m_{2}^{1}=\frac{1}{h}-\frac{a}{2}
$$

Substituting $m_{2}^{1}$ by its expression we obtain:

$$
m_{1}^{1}=\frac{12(a-1)}{S^{3}} \frac{\varepsilon^{3}}{h^{3}}-\frac{6}{S} \frac{\varepsilon^{3}}{h^{2}} .
$$

Back to $M_{1}^{1}$ and $M_{2}^{1}$ we get:

$$
\left\{\begin{array}{l}
M_{1}^{1}=\frac{a+1}{S^{3}} \frac{\varepsilon}{h}-\frac{6 a}{S^{3}} \frac{\varepsilon^{3}}{h^{2}}+\frac{12 a(a-1)}{S^{5}} \frac{\varepsilon^{3}}{h^{3}} \\
M_{2}^{1}=-\frac{(a+1) a}{S^{3}} \frac{\varepsilon}{h}-\frac{6}{S^{3}} \frac{\varepsilon^{3}}{h^{2}}+\frac{12(a-1)}{S^{5}} \frac{\varepsilon^{3}}{h^{3}}
\end{array}\right.
$$

Finally, we obtain the exact expressions of bubble basis functions $p_{b}^{1}=\left(p_{b 1}^{1}, p_{b 2}^{1}\right)$ and $\Psi_{b}^{1}=\left(\beta_{b}^{1}, \gamma_{b}^{1}, \theta_{b}^{1}, \eta_{b}^{1}\right)$ by substituting in (60) to (63) the constants $m_{1}^{1}, m_{2}^{1}, M_{1}^{1}$ and $M_{2}^{1}$ by their values respectively. Similarly working as in case $(i)$ we have for case $(i i)$ :

$$
\left\{\begin{array}{l}
\eta_{b}^{2}(\xi)=-1+\frac{\xi}{h}+\frac{m_{2}^{2}}{\varepsilon S} \\
\theta_{b}^{2}(\xi)=\frac{S m_{1}^{2}}{2 \varepsilon^{3}} \xi(h-\xi)
\end{array}\right.
$$


For the expressions of $\beta_{b}^{2}$ and $\gamma_{b}^{2}$ we get (respectively):

$$
\begin{aligned}
& \beta_{b}^{2}(\xi)=-\frac{a S}{6 \varepsilon^{3}} m_{1}^{2} \xi^{3}+\left(\frac{a S m_{1}^{2} h}{4 \varepsilon^{3}}-\frac{a}{2 h}\right) \xi^{2}+\left(\frac{m_{2}^{2}}{\varepsilon S}+\frac{1}{h}+a\right) \xi \\
& \gamma_{b}^{2}(\xi)=\frac{S}{6 \varepsilon^{3}} m_{1}^{2} \xi^{3}-\left(\frac{S m_{1}^{2} h}{4 \varepsilon^{3}}-\frac{1}{2 h}\right) \xi^{2}+\left(\frac{a m_{2}^{2}}{\varepsilon S}+\frac{1}{h}-1\right) \xi
\end{aligned}
$$

in other words, we have:

$$
\gamma_{b}^{2}(\xi)=-\frac{\beta_{b}^{2}(\xi)}{a}+\left(\frac{a+1}{a h}+\frac{a+1}{a S \varepsilon} m_{2}^{2}\right) \xi
$$

We then determine the constants $m_{1}^{2}$ and $m_{2}^{2}$ by using the conditions $\beta_{b}^{2}(h)=\gamma_{b}^{2}(h)=0$. Hence, according to $(69)$ one has

$$
m_{2}^{2}=S \frac{\varepsilon}{h}
$$

Using the expression of $\beta_{b}^{2}(\xi)$ given by $(69)$ and recalling $\beta_{b}^{2}(h)=0$ yields:

$$
\frac{a S}{12} m_{1}^{2} \frac{h^{2}}{\varepsilon^{3}}+\frac{1}{\varepsilon S} m_{2}^{2}=-\frac{1}{h}-\frac{a}{2} .
$$

Replacing $m_{2}^{2}$ by its value gives:

$$
m_{1}^{2}=-\frac{6}{S}\left(\frac{4}{a}+h\right) \frac{\varepsilon^{3}}{h^{3}}
$$

Consequently:

$$
M_{1}^{2}=-\frac{6}{S^{3}}(4+a h) \frac{\varepsilon^{3}}{h^{3}}+\frac{1}{S} \frac{\varepsilon}{h} ; \quad M_{2}^{2}=-\frac{6}{S^{3}}\left(\frac{4}{a}+h\right) \frac{\varepsilon^{3}}{h^{3}}-\frac{a}{S} \frac{\varepsilon}{h} .
$$

Finally, we obtain the expressions of $p_{b}^{2}=\left(p_{b 1}^{2}, p_{b 2}^{2}\right)$ and $\Psi_{b}^{2}=\left(\beta_{b}^{2}, \gamma_{b}^{2}, \theta_{b}^{2}, \eta_{b}^{2}\right)$ by replacing the constants $m_{1}^{2}, m_{2}^{2}, M_{1}^{2}$ and $M_{2}^{2}$ by their values respectively.

Application to the perturbed problem. Consider the problem $\left(P_{h}\right)$, if we select $\tau_{h}=\tau_{l}$ in the first equation and $q_{h}=q_{l}$ in the second one, we get:

$$
\left\{\begin{array}{l}
a_{h}\left(w_{l}, \tau_{l}\right)+b_{h}\left(\tau_{l}, p_{l}\right)+a_{h}\left(w_{b}, \tau_{l}\right)+b_{h}\left(\tau_{l}, p_{b}\right)=l_{h}\left(\tau_{l}\right) \\
b_{h}\left(w_{l}, q_{l}\right)+b_{h}\left(w_{b}, q_{l}\right)=0 .
\end{array}\right.
$$


Where:

$$
\begin{aligned}
& a_{h}\left(w_{l}, \tau_{l}\right)=\varepsilon \Sigma_{K} S_{K} \int_{K} \eta_{l} \underline{\eta}_{l} \mathrm{~d} x+\frac{\varepsilon^{3}}{12} \Sigma_{K} \frac{1}{S_{K}} \int_{K} \theta_{l}^{\prime} \underline{\theta}_{l}^{\prime} \mathrm{d} x \\
& b_{h}\left(\tau_{l}, p_{l}\right)=\Sigma_{K}\left\{\left(\underline{\beta}_{l}^{\prime}-a_{k} \underline{\theta}_{l}-\underline{\eta}_{l}, p_{l 1}\right)_{K}+\left(\underline{\gamma}_{l}^{\prime}+\underline{\theta}_{l}-a_{K} \underline{\eta}_{l}, p_{l 2}\right)_{K}\right\} \\
& b_{h}\left(\tau_{l}, p_{b}\right)=\Sigma_{K}\left\{\left(\underline{\beta}_{l}^{\prime}-a_{K} \underline{\theta}_{l}-\underline{\eta}_{l}, p_{b 1}\right)_{K}+\left(\underline{\gamma}_{l}^{\prime}+\underline{\theta}_{l}-a_{K} \underline{\eta}_{l}, p_{b 2}\right)_{K}\right\} \\
& a_{h}\left(w_{b}, \tau_{l}\right)=\varepsilon \Sigma_{K} S_{K} \int_{K} \eta_{b} \underline{\eta}_{l} \mathrm{~d} x \\
& b_{h}\left(w_{b}, q_{l}\right)=\Sigma_{K}\left\{\left(\beta_{b}^{\prime}-a_{K} \theta_{b}-\eta_{b}, q_{l 1}\right)_{K}+\left(\gamma_{b}^{\prime}+\theta_{b}-a_{K} \eta_{b}, q_{l 2}\right)_{K}\right\} .
\end{aligned}
$$

Where, integrating by parts we have used the equality:

$$
\sum_{K} \frac{1}{S_{K}} \int_{K} \theta_{b}^{\prime} \underline{\theta_{l}^{\prime}} \mathrm{d} x=\sum_{K} \frac{1}{S_{K}}\left[\left(\theta_{b}, \underline{\theta_{l}^{\prime}}\right)_{\partial K}-\left(\theta_{b}, \underline{\theta_{\ell}^{\prime \prime}}\right)_{K}\right]=0
$$

Now, writing:

$$
w_{b}=\sum_{j=1}^{n_{K}} c_{j, K}\left(\beta_{b}^{j}, \gamma_{b}^{j}, \theta_{b}^{j}, \eta_{b}^{j}\right)+\left(\beta_{b f}, \gamma_{b f}, \theta_{b f}, \eta_{b f}\right) ; \quad\left(p_{b 1}, p_{b 2}\right)=\sum_{j=1}^{n_{K}} d_{j, K}\left(p_{b 1}^{j}, p_{b 2}^{j}\right)+\left(p_{f 1}, p_{f 2}\right)
$$

If we substitute in (72) the expressions of $\beta_{b}^{j}, \gamma_{b}^{j}, \theta_{b}^{j}, \eta_{b}^{j}$ and in (75) those of $\left(p_{b 1}^{j}, p_{b 2}^{j}\right)$ and $\left(p_{f 1}, p_{f 2}\right)$, we obtain a linear system with coefficients $c_{j, K}, d_{j, K}$ as unknowns.

\subsection{Numerical experiments}

Each of the standard Galerkin $(G)$ and the Galerkin with bubbles $(G+B F)$ leads to the matricial formulation:

$$
(S)\left\{\begin{array}{l}
A \underline{X}+B^{\perp} \underline{P}=\underline{F} \\
B \underline{X}=\underline{G}
\end{array}\right.
$$

where $\underline{X}$ and $\underline{P}$ are vectors which components are the nodal parameters of displacement and Lagrange multipliers respectively, $A$ and $B$ are the matrices corresponding to the bilinear forms $a(.,$.$) and b(.,),. \underline{F}$ and $\underline{G}$ the second members. If $A_{K}$ and $B_{K}$ denote the element matrices it will be interesting to see that, with piecewise linear elements, $A_{K}$ is bloc diagonal where the diagonal terms are the matrices $A_{\beta}=A_{11}, A \gamma=A_{22}, A_{\theta}=A_{33}$ and $A_{\eta}=A_{44}$ associated to the components displacement vector $\beta, \gamma, \theta$ and $\eta$ respectively. Note that in the case of standard Galerkin $(G), A_{\beta}=A_{\gamma}=0$ and:

$$
A_{\theta}=\frac{1}{12\left(a^{2}+1\right)^{\frac{1}{2}}} \frac{\varepsilon^{2}}{h}\left(\begin{array}{cc}
1 & -1 \\
-1 & 1
\end{array}\right) ; \quad A \eta=\frac{\left(a^{2}+1\right)^{\frac{1}{2}}}{6} h\left(\begin{array}{ll}
2 & 1 \\
1 & 2
\end{array}\right)
$$


$B$ is of the form, with $\left(B_{21}=B_{12}=0, B_{13}=B_{24}=a B_{14}\right)$ and with $B_{11}=B_{22}=\frac{1}{2}\left(\begin{array}{cc}-1 & 1 \\ -1 & 1\end{array}\right)$; $B_{14}=-B_{23}=-\frac{h}{6}\left(\begin{array}{cc}2 & 1 \\ 1 & 2\end{array}\right)$

$$
B=\left[\begin{array}{llll}
B_{11} & B_{12} & B_{13} & B_{14} \\
B_{21} & B_{22} & B_{23} & B_{24}
\end{array}\right] .
$$

In the Galerkin with bubbles method the matrix $A$ becomes $A+b(A)$, the the diagonal terms are now:

$$
A_{\beta}+b\left(A_{\beta}\right), A_{\gamma}+b\left(A_{\gamma}\right), A_{\theta}+b\left(A_{\theta}\right) \text { and } A \eta+b\left(A_{\eta}\right)
$$

where $b\left(A_{\beta}\right)=b\left(A_{\gamma}\right)=b\left(A_{\theta}\right)=0$ and $b\left(A_{\eta}\right)=\left(\begin{array}{cc}\frac{1-h}{2} & 0 \\ 0 & -\frac{h}{3}+\frac{1}{2} \frac{a+1}{a^{2}+1}\end{array}\right)$ and similarly the matrix $B$ becomes $B+b(B)$ with $b\left(B_{12}\right)=b\left(B_{21}\right)=0, b\left(B_{14}\right)=-\frac{b\left(B_{13}\right)}{a}, b\left(B_{24}\right)=-a b\left(B_{23}\right)$ and:

$$
\begin{array}{ll}
b\left(B_{11}\right)=\left(\begin{array}{cc}
\frac{1}{2}-M_{1}^{1} & 0 \\
0 & \frac{1}{2}+M_{1}^{2}
\end{array}\right) ; & b\left(B_{13}\right)=\left(\begin{array}{cc}
a h\left(\frac{1}{3}-\frac{M_{1}^{1}}{2}\right) & 0 \\
0 & a h\left(\frac{1}{3}-\frac{M_{1}^{2}}{2}\right)
\end{array}\right) \\
b\left(B_{22}\right)=\left(\begin{array}{cc}
-\frac{1}{2}+M_{2}^{1} & 0 \\
0 & \frac{1}{2}-M_{2}^{2}
\end{array}\right) ; & b\left(B_{23}\right)=\left(\begin{array}{cc}
-\frac{h}{3}+M_{2}^{1} \frac{h}{2} & 0 \\
0 & -\frac{h}{3}+M_{2}^{2} \frac{h}{2}
\end{array}\right) .
\end{array}
$$

To illustrate the results above, we test the methods on an arch of shape $\Phi(x)=x^{2}-x$ subjected to uniform unit load.

\subsection{Conclusion}

A standard Galerkin mixed method $(\mathrm{G})$ and the Galerkin method enriched with residual-free bubbles (RFB) for equal order linear elements are considered to approximate the displacement of a general arch problem. We show that the (RFB) method consists in fact of the standard Galerkin formulation plus mesh dependent terms. The bubble functions, assumed to satisfy the governing differential equations in each element interior of a regular grid, are determined exactly. Numerical experiments are carried out for an arch with a given smooth shape which supports a uniform unit load. The results are presented for the values $\varepsilon=1, \varepsilon=10^{-1}$ and $\varepsilon=10^{-2}$ of the thickness. The graphics show that the approximated rotation of the normal vector by the Galerkin method has a similar behaviour to exact one (Figs. 1 and 2). On the other hand, the approached membrane energy with classical Galerkin method presents a pathological behaviour which consists of a boundary layer phenomena observed for $\frac{\varepsilon}{h} \leq 10^{-1}$. Experiments show that an improvement is brought to the behaviour of the membrane energy component by the use of the (RFB) method (Figs. 3 and 4). 


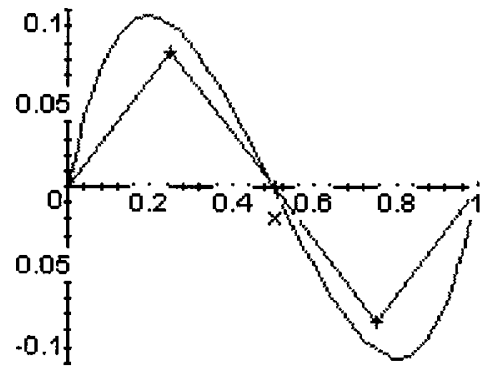

(a) eps $=1 \quad \mathrm{~h}=0.25$

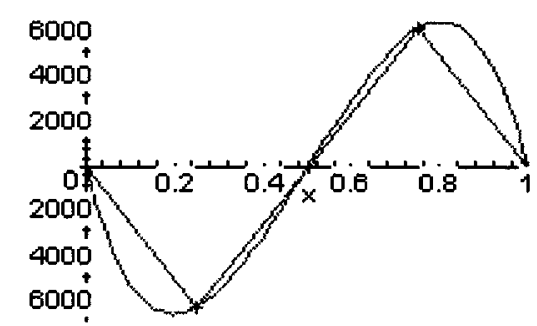

(c) eps $=0.0100 \quad \mathrm{~h}=0.25$

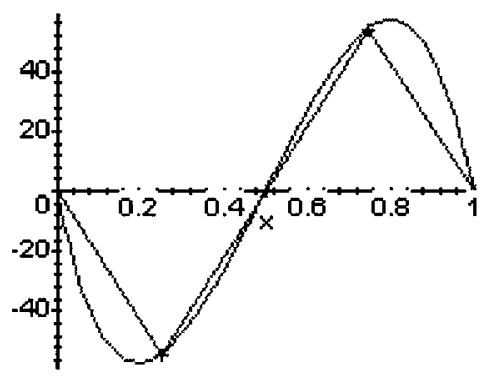

(b) eps $=0.100 \quad h=0.25$

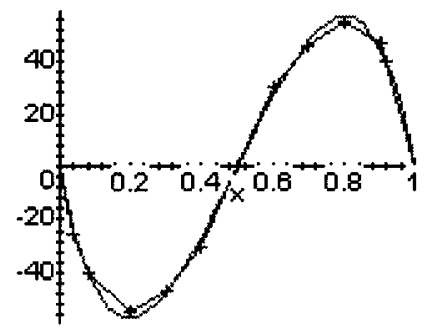

(d) eps $=1 \quad \mathrm{~h}=0.100$

FIGURE 1. Standard Galerkin method with piecewise linear elements.

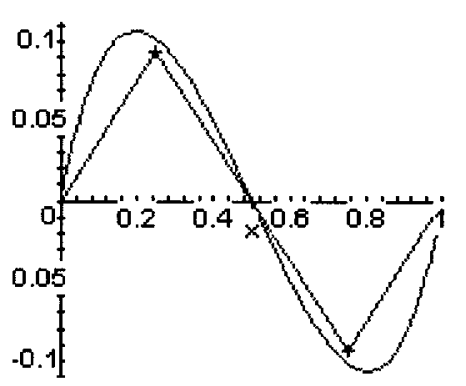

(a) eps $=1 \quad h=0.25$

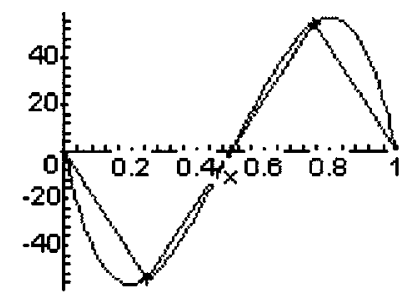

(b) eps $=0.100 \quad \mathrm{~h}=0.25$

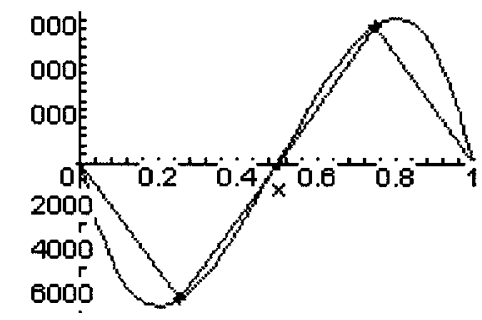

(c) eps $=0.0100 \quad \mathrm{~h}=0.25$

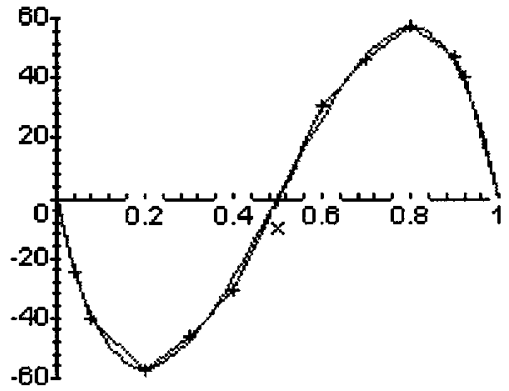

(d) eps=1 $h=0.100$

Figure 2. Galerkin+bubbles method. 


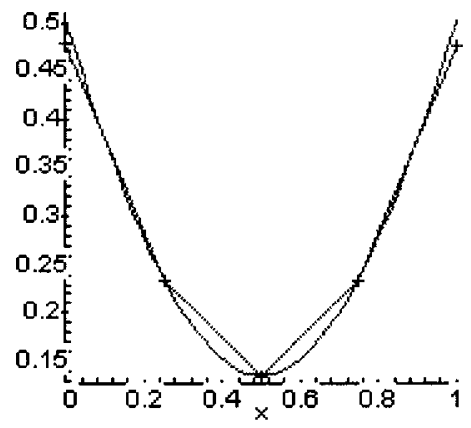

(a) eps $=1 \quad h=0.25$

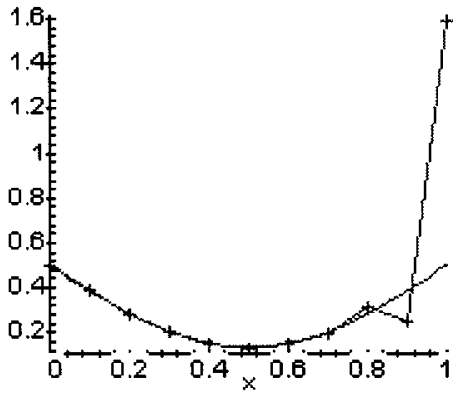

(c) eps $=0.0100 \quad \mathrm{~h}=0.25$

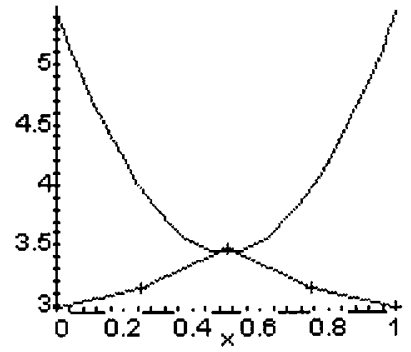

(b) eps $=0.100 \quad h=0.25$

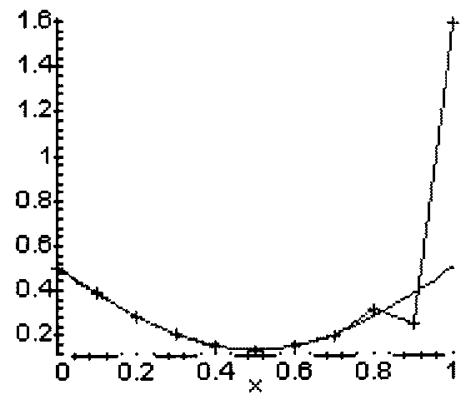

(d) eps $=1 \quad h=0.100$

FIgURE 3. Standard Galerkin method with piecewise linear elements.

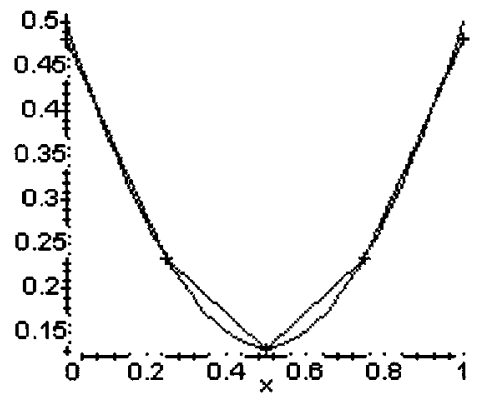

(a) eps $=1 \quad h=0.25$

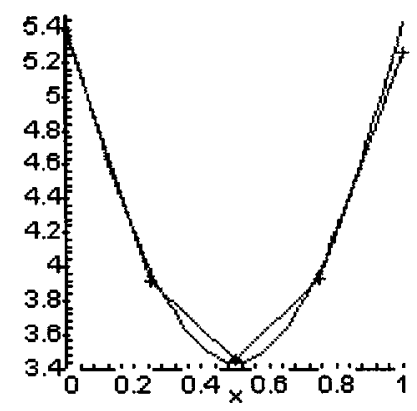

(c) eps $=0.0100 \quad \mathrm{~h}=0.25$

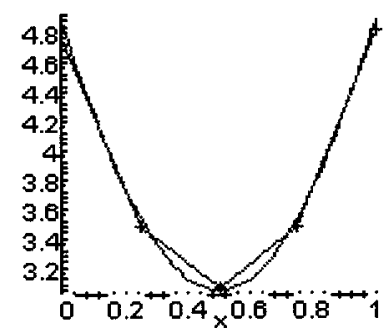

(b) eps $=0.100 \quad \mathrm{~h}=0.25$

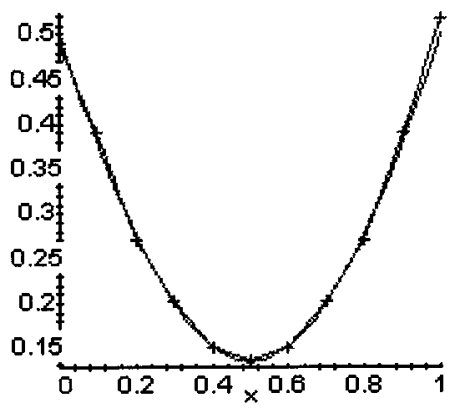

(d) eps $=1 \quad h=0.100$

FiguRE 4. Galerkin+bubbles method. 


\section{REFERENCES}

[1] D.N. Arnold and R.S. Falk, A uniformly accurate finite element method for the Reissner Mindlin plate. SIAM J. Numer. Anal 26 (1989) 1276-1250.

[2] I. Babuska, The finite element method with Lagrangian multipliers. Numer. Math 20 (1973) 179-192.

[3] I. Babuska and M. Suri, On the locking and robustness in the finite element method. SIAM J. Numer. Anal. 29 (1992) $1276-1290$.

[4] C. Baiocchi, F. Brezzi and L. Franca, Virtual bubbles and the Galerkin-Least-squares method. Comput. Methods Appl. Mech. Engrg. 105 (1993) 125-141.

[5] M. Bernadou and Y. Ducatel, Approximation of a general arch problems by straight beam elements. Numer. Math. 40 (1982) $1-29$.

[6] F. Brezzi, On the existence, uniqueness and approximation of saddle-point problems arising from Lagrange multipliers. RAIROAnal. Numér. (1974) 129-151.

[7] F. Brezzi and I. Douglas, Stabilized mixed methods for the stokes problem. Numér. Math. 53 (1988) 225-236.

[8] F. Brezzi and M. Fortin, Mixed and hybrid finite Element Methods. Springer-Verlag, Berlin, New-York, Springer Ser. Comput. Math. 15 (1991).

[9] F. Brezzi and A. Russo, Choosing bubbles for advection-diffusion problems. Math. Models Methods Appl. Sci. 4 (1994) $571-578$.

[10] B. Budiansky and J.L. Sanders, On the best first order linear shell theory. Progr. Appl. Mech., Mac Millan, New-York, 129-140.

[11] D. Chenais, Rousselet and B. Benedict, Design sensibivity for arch structures with respect to midsurface shape under static loading. J. Optim. Theory Appl. 58 (1988) 225-239.

[12] D. Chenais and J.-C. Paumier, On the locking phenomenon for a class of elliptic problems. Numer. Math. 67 (1994) $427-440$

[13] P.G. Ciarlet, The finite element method for elliptic problems. North Holland, Amsterdam (1978).

[14] Ph. Destuyender, Some numerical aspects of mixed finite elements for bending plates. Comput. Methods. Appl. Mech. Engrg. 78 (1990) 73-87.

[15] L.P. Franca and T.J.R. Hughes, Two classes of mixed finite element methods. Comput. Methods Appl. Mech. Engrg. 69 (1986) 89-129.

[16] L.P. Franca and A. Russo, Unlocking with residual-free bubbles. Comput. Methods Appl. Mech. Engrg. 142 (1997) 361-364

[17] A. Habbal and D. Chenais, Deterioration of a finite element method for arch structures when thickness goes to zero. Numer. Math. 62 (1992) 321-341.

[18] V. Lods, A new formulation for arch structures. Application to optimization problems. RAIRO-Modél. Math. Anal. Numér. 28 (1994) 873-902.

[19] A.F.D. Loula, L.P. Franca, T.J.R. Hughes and I. Miranda, Stability Convergence and accuracy of a New finite element method for the circular arch problem. Comput. Methods Appl. Mech. Engrg. 63 (1987) 281-303.

[20] Z. Ould Zeidane, Contributions théoriques en Optimisation et Modélisation des structures. Thèse Université de Nice SophiaAntipolis, Nice (1995).

[21] A. Russo, Residual-free bubbles and Stabilized methods, in Proc. of the ninth International Conference on finite Elements in Fluids-New Trends and Applications, M.M. Cacchi, K. Morgan, J. Pariaux, B.A. Schreffer, O.C. Zienkiewicz, Eds., Venice (1995) 377-386.

[22] A. Russo, Bubble Stabilization of finite element methods for the linearized incompressible Navier-Stokes equations. Comput. Methods Appl. Mech. Engrg. 132 (1996) 333-343.

To access this journal online:

www.edpsciences.org 